\title{
Ultrastructure Study of the Spermiogenesis in the Aphanius dispar (Rüppell 1828) (Pisces: Cyprinodontidae)
}

\author{
Taher A. Ba-Omar \\ Biology Department, College of Science, Sultan Qaboos University, P.O.Box 36 \\ Muscat 123, OMAN
}

Aphanius dispar (Rüppell 1828), a cyprinodont fish, is common and widely distributed in the fresh and brackish waters of the Sultanate of Oman. It is eurytopic and reproductively very successful, capable of building high population densities within a few months.

Male Aphanius dispar fish were collected and were kept in an aquarium within the Department of Biology Laboratory, Sultan Qaboos University, Oman. The fish were sacrificed and the testes were dissected out and were immediately removed and fixed in Karnovsky fixative buffered with sodium cacodylate to a $\mathrm{pH}$ of 7.4 for four hours and then cut into small pieces. The tissue were washed in cacodylate buffer and then post-fixed in $1 \%$ aqueous solution of osmium tetroxide for 1 hour and dehydrated in a series of alcohol before embedding in Agar 100 resin. Semi-thin and ultrathin sections were cut using Reichert ultramicrotome. The semi-thin sections were stained with toluidine blue and the ultra-thin sections were stained with uranyl acetate and poststained in lead citrate. The sections were examined using a Zeiss EM900 TEM.

Testes are made up of several cysts (Fig. 1). Each cyst is composed of several germ cells at the same stage of development (Fig. 1). Spermiogenesis took place within each cyst. It starts when early spermatids start to lose contact with each other (Fig.2,3). The early spermatids possess spherical nuclei with dense chromatin. Other cytoplasmic organelles such as mitochondria, ribosomes and Golgi are also present (Fig. 3, 4). Centrioles were centrally located in the area where the head of spermatozoa is going to take place (Fig.3,4). Mitochondria are seen in good number and seen in association with the centrioles (Fig.3, 4). In late spermatids, nuclei were seen to be condensed and had kidney shaped. The nuclei are now seen eccentric at one pole of cell (Fig. 2). The flagella show 9 doublets and 2 microtubules (Fig.5). Fins were also present within the flagella (Fig. 6). 


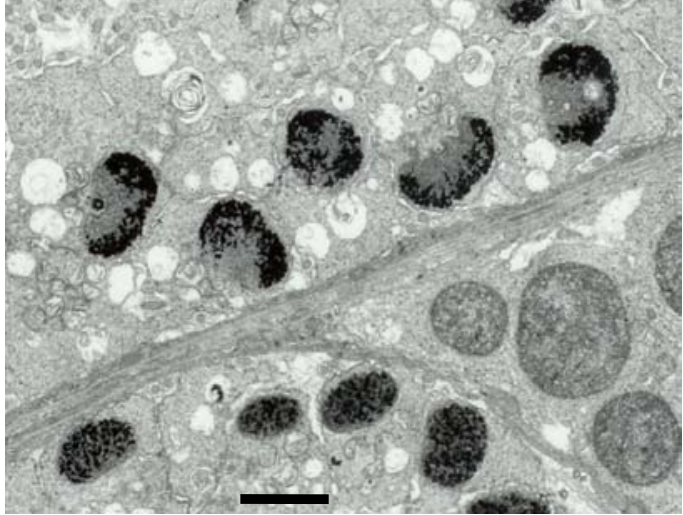

Fig. 1

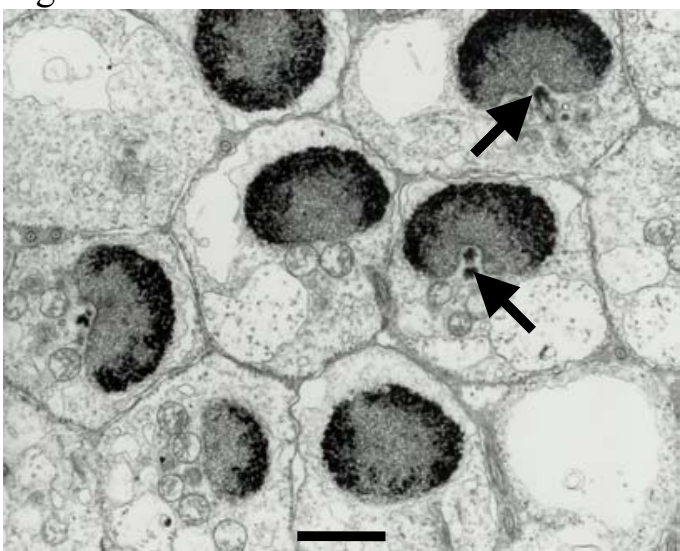

Fig. 3

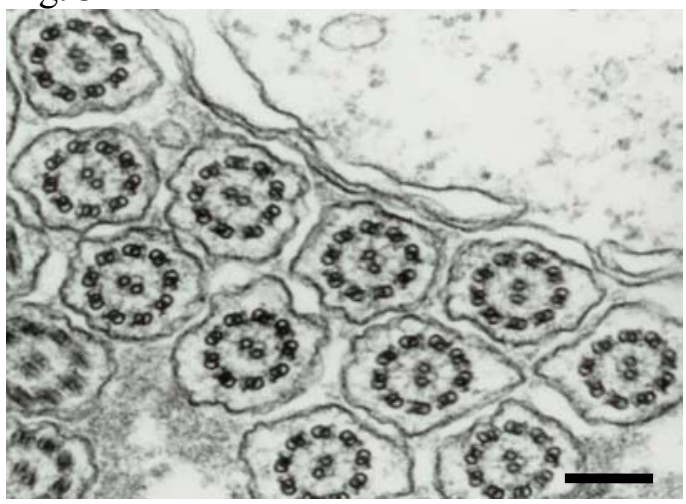

Fig. 5

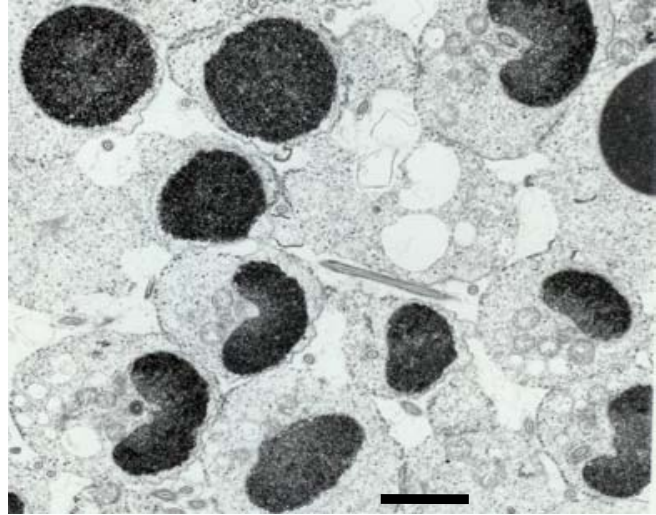

Fig. 2

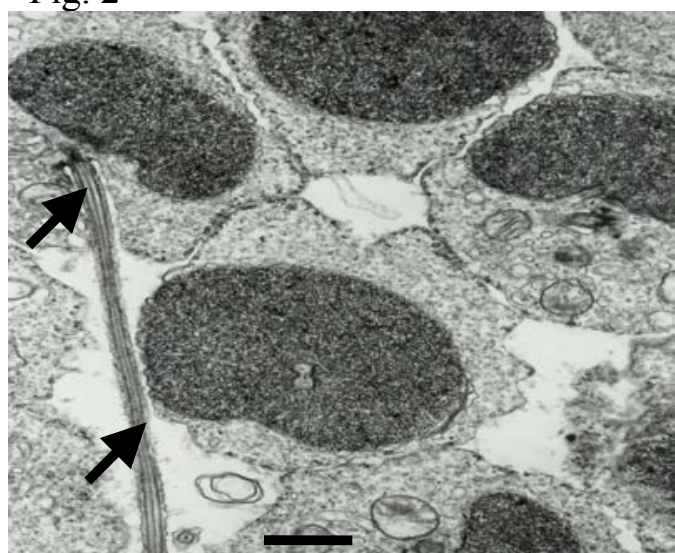

Fig. 4

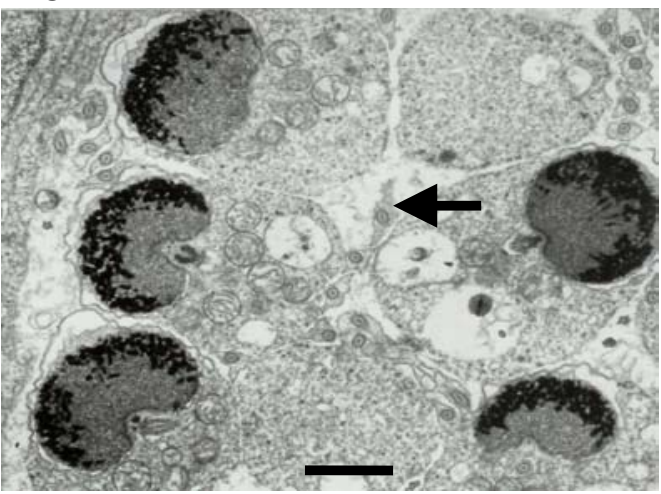

Fig. 6

Fig.1. Shows germ cells at different cysts at different developmental stages. Bar $=1.7 \mu \mathrm{m}$

Fig. 2. Shows germ cells losing contact with their neighbors and some nuclei with kidney - shaped. Appearance. Bar $=2.5 \mu \mathrm{m}$

Fig. 3. Shows the presence of cytoplasmic organelles such as mitochondria which are associated with centrioles (arrows). Bar $=1 \mu \mathrm{m}$

Fig. 4. Shows germ cells with organelles such flagella (arrows) . Bar $=1.2 \mu \mathrm{m}$

Fig. 5. Shows several flagellae with $9+2$ micrtotubles.Bar $=0.2 \mu \mathrm{m}$

Fig. 6. Shows some nuclei with kidney - shaped Appearance and flagella with fins (arrows). Bar $=1.7 \mu \mathrm{m}$ 